\title{
Erratum to: The Economics of Self-Destructive Choices
}

Erratum to:

Chapter 5 in: S. Ikeda, The Economics of Self-Destructive Choices, Advances in Japanese Business and Economics

DOI 10.1007/978-4-431-55793-7_5

The publisher regrets that Fig. 5.1 was inadvertently reproduced incorrectly during the publication process. The incorrect image has now has been replaced. The inappropriate text layout/style in the print version p.145-146 has also been corrected.

The updated original online version for this chapter can be found at DOI 10.1007/978-4-431-55793-7_5 